\title{
ALGUNOS INVARIANTES TOPOLOGICOS DE FOLIACIONES POR CURVAS EN LA VECINDAD DE UNA SINGULARIDAD AISLADA
}

\author{
Renato Benazic Tomé $e^{1}$
}

RESUMEN.- En el presente artículo, proporcionamos algunos invariantes topológicos de foliaciones analíticas por curvas definidas en una variedad compleja de dimensión 3 en la vecindad de una singularidad dicrítica aislada.

\section{INTRODUCCION}

La finalidad de esta sección es dar algunas definiciones y establecer las notaciones necesarias útiles para el desarrollo del presente trabajo.

Sea $\mathcal{M}^{n}$ una variedad analítica compleja de dimensión $n$ y consideremos en ella una foliación analítica singular por curvas. Esto significa que en cualquier punto $p \in \mathcal{M}^{n}$, la foliación es generada por el campo vectorial holomorfo:

$$
Z=\sum_{i=1}^{n} Z_{i} \frac{\partial}{\partial z_{i}}, Z_{1}, Z_{2}, \ldots, Z_{n} \in \mathcal{O}_{n, p}
$$

y m.c.d. $\left(Z_{1}, Z_{2}, \ldots, Z_{n}\right)=1$, donde $\mathcal{O}_{n, p}$ es el anillo de gérmenes de las funciones analíticas en $p$. En lo sucesivo, denotaremos por $\mathcal{F}_{Z}$ a esta foliaciación, diremos que el campo $Z$ genera la foliación $\mathcal{F}_{Z}$ y las funciones $Z_{i}$ serán llamadas componentes o coordenadas de $Z$. El lector interesado en conocer detalles de la teoría de funciones analíticas de variables complejas, deberá consultar [10] y para los que deseen profundizar en la teoría de las variedades analíticas complejas, recomendamos [13]. Sea $p \in \mathcal{M}^{n}$ y consideremos una carta $(U, \phi)$ de $\mathcal{M}^{n}$ alrededor del punto $p$ tal que $\phi(p)=0 \in \mathbb{C}^{n}$, claramente $Z_{i} \circ \phi^{-1}$ es una función analítica de varias variables complejas definida en una vecindad del origen y por lo tanto, ella tiene un desarrollo en series de potencias

$$
Z_{i} \circ \phi^{-1}=\sum_{k \geq 0} Z_{i}^{k}, \quad 1 \leq i \leq n,
$$

donde los $Z_{i}^{k}$ son polinomios homogéneos de grado $k$ en $n$ variables complejas. El orden de $Z_{i} \circ \phi^{-1}$ en el $0 \in \mathbb{C}^{n}$ es, por definición, el menor número entero $v_{i}$ tal que $Z_{i}^{k} \equiv 0$, para todo $k<v_{i}$ y $A_{i}^{v_{i}} \not \geq 0$. No es difícil probar que el número

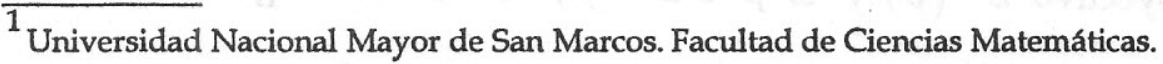


$v_{i}$ es independiente de la elección de la carta $(U, \phi)$, por esta razón el entero $v_{i}$ es llamado el orden de $Z_{i}$ en $p$ y lo denotamos por $\operatorname{ord}_{p}\left(Z_{i}\right)$. La multiplicidad algebraica de la foliación $\mathcal{F}_{Z}$ (o del campo $Z$ ) en el punto $p \in \mathcal{M}^{n}$, denotada por $m_{p}\left(\mathcal{F}_{Z}\right)$ (o simplemente por $m_{p}\left(\mathcal{F}_{Z}\right)$ ), es definida como el mínimo de los órdenes $\operatorname{ord}_{p}\left(Z_{i}\right)$. Un punto $p \in \mathcal{M}^{n}$ es llamado punto singular de la foliación $\mathcal{F}_{Z}$ (o del campo $Z$ ) si y sólo si $m_{p}(Z) \geq 1$, en caso contrario, decimos que $p$ es un punto regular. El conjunto de todos los puntos singulares de la foliación $\mathcal{F}_{Z}$ será denotado por $\operatorname{Sing}\left(\mathcal{F}_{Z}\right)$. Un purnto $p \in \mathcal{M}^{n}$ es llamado singularidad aislada de $Z$ si y sólo si $p \in \operatorname{sing} \mathcal{F}_{Z}$ y existe una vecindad abierta $U \subseteq \mathcal{M}^{n}$ de $p$ tal que todos los elementos de $U-\{p\}$ son puntos regulares de $Z$.

Un punto $p \in \mathcal{M}^{n}$ es llamado irreducible su y sólo si $m_{p}(Z)=1$ y la parte lineal de $Z$ en $p$ (i. e. $D Z(p)$ ) tiene al menos un autovalor no nulo.

Sea $E: \tilde{\mathcal{M}}^{n} \rightarrow \mathcal{M}^{n}$ el blow-up centrado en el punto $p \in \operatorname{Sing}\left(\mathcal{F}_{Z}\right)$. Entonces existe una única manera de extender $E^{*}\left(F_{Z}-\{p\}\right)$ a una foliación analítica singular $\tilde{\mathcal{F}}_{Z}$ sobre una vecindad del espacio proyectivo $\mathbb{C} P(n-1)=E^{-1}(p) \subset \tilde{\mathcal{M}}^{n}$, con conjunto singular de codimensión mayor o igual a 2. En este caso decimos que $\tilde{\mathcal{F}}_{Z}$ es el transformado estricto de $\mathcal{F}_{Z}$ por $E$. El lector interesado en mayores detalles sobre el tema, puede consultar las referencias [9], [12], [3] y [7].

Decimos que $p$ es una singularidad no dicrítica de $\mathcal{F}_{Z}$ si y sólo si $E^{-1}(p)$ es invariante por $\tilde{\mathcal{F}}_{z}$, es decir, $E^{-1}(p)$ es unión de hojas y singularidades de $\tilde{\mathcal{F}}_{z}$. En caso contrario, $p$ es llamado singularidad dicrítica. El conjunto de las foliaciones analíticas por curvas sobre $\mathcal{M}^{n}$ con una singularidad dicrítica aislada, será denotado por $\mathcal{D}^{n}$.

Sea $\mathcal{F}_{Z} \in \mathcal{D}^{n}$ generada por el campo vectorial holomorfo $Z$, tal que $m_{p}(Z)=v$. En [1] se demuestra que es posible asociar a $J_{p}^{v}(Z)$, el primer jet no nulo de $\mathcal{F}_{Z}$ en $p$, un polinomio homogéneo $P_{v-1}$ de $n$ variables de grado $v-1$, tal que:

$$
J_{p}^{\nu}(Z)=P_{\nu-1} R
$$

donde $R\left(z_{1}, \ldots, z_{n}\right)=\sum_{i=1}^{n} z_{i} \frac{\partial}{\partial z_{i}}$ es el campo radial.

El polinomio $P_{v-1}$ define una hipersuperficie algebraica $S$ sobre el espacio proyectivo $E^{-1}(p)=\mathbb{C} P(n-1)$ :

$$
S=\left\{\left[z_{1} ; \ldots ; z_{n}\right] \in \mathbb{C} P(n-1): P_{\nu-1}\left(z_{1}, \ldots, z_{n}\right)=0\right\}
$$

Observe que $S$ contiene todas las singularidades del transformado estricto $\tilde{\mathcal{F}}_{Z}$. La hipersuperficie $S$ tiene la siguiente interpretación geométrica. $\mathrm{Si}$ $\tilde{p} \in S-\operatorname{Sing}\left(\tilde{\mathcal{F}}_{Z}\right)$ entonces la hoja $\tilde{L}$ de $\tilde{\mathcal{F}}_{Z}$ que pasa por $\tilde{p}$ es tangente al espacio proyectivo $E^{-1}(p)$ y si $\tilde{p} \in E^{-1}(p)-S$ entonces $\tilde{L}$ es tranversal a $E^{-1}(p)$. 
Un caso importante ocurre cuando $\operatorname{Sing}\left(\tilde{\mathcal{F}}_{Z}\right)=\emptyset$, es decir, cuando el transformado estricto de $\mathcal{F}_{Z}$ no tiene singularidades en $\tilde{\mathcal{M}}^{n}$. Denotaremos por $\mathcal{D}_{0}^{n}$ al conjunto de tales foliaciones. Observe que, en este caso, cada $\tilde{p} \in E^{-1}(p)$ es un punto regular de $\tilde{\mathcal{F}}_{Z}$. Por lo tanto es posible definir el indice de intersección $i_{\tilde{p}}\left(E^{-1}(p), L\right)$ entre la hoja $\tilde{L}$ de $\tilde{\mathcal{F}}_{z}$ que pasa por $\tilde{p} \in E^{-1}(p)$ y el espacio proyectivo $E^{-1}(p)$. El índice de intersección tiene la siguiente propiedad:

$$
i_{\tilde{p}}\left(E^{-1}(p), \tilde{L}\right)>1 \Leftrightarrow \tilde{L} \text { es tangente a } E^{-1}(p) \Leftrightarrow \tilde{p} \in S .
$$

El objetivo principal del presente trabajo, es estudiar los invariantes topológicos de las foliaciones $\mathcal{F}_{Z} \in \mathcal{D}_{0}^{n}$ cuando $n=3$.

\section{ALGUNOS RESULTADOS PRELIMINARES}

Sea $\mathcal{F}_{Z} \in \mathcal{D}_{0}^{n}$ generado por el campo vectorial holomorfo $Z$, sin pérdida de generalidad, podemos suponer que la singularidad aislada dicrítica es el $0 \in \mathbb{C}^{n}$. Sabemos que cualquier punto $\tilde{p} \in E^{-1}(p)=\mathbb{C} P(n-1)$ es un punto regular de la foliación $\tilde{\mathcal{F}}_{Z}$. Sea $\tilde{L}$ la hoja de $\tilde{\mathcal{F}}_{Z}$ que pasa por $\tilde{p}$. Desde que $E^{-1}(0)$ y $\tilde{L}$ son subvariedades de $\mathbb{C}^{n}$ de dimensiones complementarias (ver [8]) y $\tilde{p} \in E^{-1}(0) \cap \tilde{L}$, podemos definir el indice de inteersección $i_{\tilde{p}}\left(E^{-1}(0), \tilde{L}\right)$ de la hoja $\tilde{L}$ con el espacio proyectivo $E^{-1}(0)$ en el punto $\tilde{p}$. En efecto, podemos suponer sin pérdida de generalidad, que $\tilde{p}$ está en la carta de blow-up en donde $E$ se expresa como:

$$
E\left(y_{1}, \ldots, y_{n}\right)=\left(y_{1} y_{n}, \ldots, y_{n-1} y_{n}, y_{n}\right)=\left(z_{1}, \ldots, z_{n}\right)
$$

Desde que $\tilde{p}=\left(y_{1}^{0}, \ldots, y_{n-1}^{0}, 0\right)$ es un punto regular, la hoja $\tilde{L}$ puede ser localmente parametrizada por una función analítica

$$
\tilde{\alpha}=\left(\tilde{\alpha}_{1}, \ldots, \tilde{\alpha}_{n}\right):\left(\mathbb{D}_{\in}, 0\right) \rightarrow(\tilde{L}, \tilde{p})
$$

donde $\mathbb{D}_{\epsilon}=\{T \in \mathbb{C}:|T|<\varepsilon\}$, tal que:

$$
\mid \begin{aligned}
& \tilde{\alpha}^{\prime}(T)=\tilde{Z}(\tilde{\alpha}(T)), \quad \forall T \in \mathbb{D}_{\epsilon} \\
& \tilde{\alpha}^{\prime}(0)=\tilde{p} .
\end{aligned}
$$

Desde que $E^{-1}(0)=\left\{y_{n}=0\right\}$, podemos definir :

$$
i_{\tilde{p}}\left(E^{-1}(0), \tilde{L}\right)=\operatorname{ord}_{0}\left(\tilde{\alpha}_{n}\right)
$$

es decir, $i_{\tilde{p}}\left(E^{-1}(0), \tilde{L}\right)=m \Leftrightarrow \tilde{\alpha}_{n}(T)=T^{m} \tilde{\varepsilon}_{n}(T)$ donde $\tilde{\varepsilon}$ es una función analítica y $\tilde{\varepsilon}_{n}(0) \neq 0$. Es claro que $i_{\tilde{p}}\left(E^{-1}(0), \tilde{L}\right) \geq 1, \quad \forall \tilde{p} \in E^{-1}(0)$. 
El índice de intersección puede ser geométricamente interpretado como el número de puntos de intersección de $\tilde{L}$ con una pequeña traslación del espacio proyectivo $\left\{y_{n}=\delta\right\}$, con $\delta \neq 0$. Para mayor información sobre las propiedades del índice de intersección entre subvariedades analíticas de dimensiones complementarias, el lector debe consultar [8].

Observe que si $\tilde{p} \in E^{-1}(0)$ entonces $\tilde{\alpha}_{n}^{\prime}(0)=P_{v-1}\left(y_{1}^{0}, \ldots, y_{n-1}^{0}, 1\right)$, concluimos que $i_{\tilde{p}}\left(E^{-1}(0), \tilde{L}\right)=1$ si sólo si $\tilde{p} \in E^{-1}(0)-S$ si y sólo si $\tilde{L}$ es transversal a $E^{-1}\left(\begin{array}{l}0 \\ 0\end{array}\right)$ en $\tilde{p}$.

Una propiedad importante es que el índice de intersección es un invariante topológico. Más específicamente, sea $Z$ un germen en $0 \in \mathbb{C}^{n}$ de un campo vectorial holomorfo y $L$ un germen en $0 \in \mathbb{C}^{n}$ de una subvariedad analítica irreducible invariante por $Z$ y de $\operatorname{dim}_{\mathbb{C}} L=1$. Si $B$ es una bola suficientemente pequeña centrada en $0 \in \mathbb{C}^{n}$ tal que $B \cap L$ es conexo, entonces $B \cap L$ es homeomorfo a un disco bidimensional $\mathbb{D}_{\epsilon}=\{T \in \mathbb{C}:|T|<\epsilon\}$. Tal homeomorfismo puede ser realizado, por ejemplo, mediante una parametrización de Puiseaux

$$
\alpha:\left(\mathbb{D}_{\epsilon}, 0\right) \rightarrow(B \cap L, 0)
$$

de $B \cap L$, donde $\alpha(T)=\left(\alpha_{1}(T), \ldots, \alpha_{n}(T)\right), \alpha_{j}(T)=T^{m_{j}} \varepsilon_{j}(T)$ con $\varepsilon_{j}(0) \neq 0$ ó $\varepsilon_{j}=0$ y $m_{j} \in \mathbb{Z}^{+}, \quad \forall 1 \leq j \leq n$.

Bajo estas condiciones, la restricción $\left.Z\right|_{B \cap L}$ puede ser considerado como germen de un campo vectorial real con singularidad aislada, luego su índice en $0 \in \mathbb{C}^{n}$ esta bien definido.

DEFINICION 1. Sean $Z, L$ y $B$ como antes. Definimos la Multiplicidad de $Z$ a lo largo de $L$ en $0, m_{0}(Z, L)$ como el índice de $\left.Z\right|_{B \cap L}$ en $0 \in \mathbb{C}^{n}$, considerado como un campo vectorial real en $B \cap L$.

La siguiente proposición, cuya demostración se encuentra en [6] establece que el número $m_{0}(Z, L)$ puede ser calculado en términos de una parametrización de Puiseaux $\alpha$ de $L$.

PROPOSICION 1. Con las notaciones anteriores, existe un único campo vectorial holomorfo $X$ en $\mathbb{D}_{\epsilon}$ con $X(0)=0$ tal que:

$$
Z(\alpha(T))=X(T) \alpha^{\prime}(T), \quad \forall T \in \mathbb{D}_{\in}
$$

Además, si $X(T)=T^{m} \beta(T) \frac{\partial}{\partial T}\left(m \in \mathbb{Z}^{+}\right)$, con $\beta(0) \neq 0$, entonces

$$
m_{0}(Z, L)=m \text {. }
$$

DEFINICION 2. Sean $\mathcal{F}_{Z}$ y $\mathcal{F}_{Z^{\prime}}$ dos gérmenes de foliaciones en $0 \in \mathbb{C}^{n}$. Decimos que $\mathcal{F}_{Z}$ y $\mathcal{F}_{Z^{\prime}}$ son topológicamente equivalentes, lo cual será denotado por $\mathcal{F}_{Z \sim \text { top }} \mathcal{F}_{Z^{\prime}}$ (o simplemente por $Z_{\sim \text { top }} Z^{\prime}$ ) si y sólo si existe $h$ germen de homeomorfismo en $0 \in \mathbb{C}^{n}$ tal que $h^{*}\left(\mathcal{F}_{Z^{\prime}}\right)=\mathcal{F}_{Z}$. Es decir, $L \in \mathcal{F}_{Z} \Rightarrow h[L] \in \mathcal{F}_{Z^{\prime}}$. El germen de homeomorfismo $h$ es llamado conjugación topológica. 
Sean $\mathcal{F}_{Z}$ y $\mathcal{F}_{Z^{\prime}}$ dos gérmenes de foliaciones con singularidad aislada en $0 \in \mathbb{C}^{n}$, tales que $\mathcal{F}_{Z \sim \text { top }} \mathcal{F}_{Z^{\prime}}$ por un homeomorfismo $h: U \rightarrow U^{\prime}$ donde $U$ y $U^{\prime}$ son vecindades abiertas del 0 . Sea $L$ una subvariedad analítica, irreducible, invariante por $Z$ con singularidad aislada en $0 \in \mathbb{C}^{n}$ y $\operatorname{dim}_{\mathbb{C}} L=1$. Podemos suponer que $L \cap U$ es conexa. Bajo éstas condiciones, se sigue que $L-\{0\}$ es una hoja de $\mathcal{F}_{Z}$ y por el teorema de Remmert-Stein (ver [10]), tenemos que $L^{\prime}=h[L]$ es una subvariedad analítica, irreducible, invariante por $Z^{\prime}$ con $\operatorname{dim}_{\mathbb{C}} L^{\prime}=1$. Por lo tanto, $m_{0}\left(Z^{\prime}, L^{\prime}\right)$ está bien definido. Tenemos el siguiente resultado.

PROPOSICION 2. La multiplicidad de $Z$ a lo largo de $\mathrm{V}$ es un invariante topológico. Más específicamente, sean $L$ y $L^{\prime}$ como antes. Si $\mathcal{F}_{Z \sim \text { top }} \mathcal{F}_{Z^{\prime}}$ entonces $m_{0}(Z, L)=m_{0}\left(Z^{\prime}, L^{\prime}\right)$.

El lector puede encontrar la prueba de la Proposición 2 en [6].

Retornando a las foliaciones dicríticas, probaremos que existe una relación entre el índice de intersección y la multiplicidad a lo largo de una subvariedad invariante. En efecto, siguiendo [2] sea $\mathcal{F}_{Z} \in \mathcal{D}_{0}^{n}$, con $m_{0}\left(\mathcal{F}_{Z}\right)=v$ y $\tilde{p} \in E^{-1}(0)=\mathbb{C} P(n-1)$, podemos suponer, sin pérdida de generalidad, que $\tilde{p}=\left(y_{1}^{0}, \ldots, y_{n-1}^{0}\right)$. Sea $\tilde{U}$ vecindad de $\tilde{p}$ tal que $\left.\tilde{L}\right|_{\tilde{U}} \cap E^{-1}(0)=\{\tilde{p}\}$, donde $\tilde{L}$ es la hoja de $\tilde{\mathcal{F}}_{Z}$ que pasa por $\tilde{p}$. Si $\varepsilon>0$ es suficientemente pequeño, entonces $\left.\tilde{L}\right|_{\tilde{U}}$ puede ser parametrizado por la función analítica

$$
\tilde{\alpha}=\left(\tilde{\alpha}_{1}, \ldots, \tilde{\alpha}_{n}\right):\left(\mathbb{D}_{\in}, 0\right) \rightarrow(\tilde{L}, \tilde{p})
$$

la cual satisface

$$
\mid \begin{aligned}
& \tilde{\alpha}^{\prime}(T)=\tilde{Z}(\tilde{\alpha}(T)), \quad \forall T \in \mathbb{D}_{\in} \\
& \tilde{\alpha}^{\prime}(0)=\tilde{p}
\end{aligned}
$$

Si $\tilde{\alpha}_{n}(T)=T^{m} \tilde{\varepsilon}_{n}(T)$ con $\tilde{\varepsilon}_{n}(0) \neq 0$ y $m \in \mathbb{Z}^{+}$, se sigue de la definición de índice de intersección que $i_{\tilde{p}}\left(E^{-1}(0), \tilde{L}\right)=m$. Sea $U$ vecindad de $0 \in \mathbb{C}^{n}$ tal que $E^{-1}[U-\{0\}] \subseteq \tilde{U}$ y $E\left[\left.\tilde{L}\right|_{\tilde{U}}-\{\tilde{p}\}\right]=L-\{0\}$. Entonces $L$ es una subvariedad analítica, irreducibłe de $U$, invariante por $\mathbb{Z}$, con singularidad aislada en 0 y $\operatorname{dim}_{\mathbb{C}} L=1$. Definimos $\alpha:\left(\mathbb{D}_{\epsilon}, 0\right) \rightarrow(L, 0)$ como:

$$
\alpha(T)=\left\{\begin{array}{lr}
E \tilde{\alpha}(T)=\tilde{Z}(\tilde{\alpha}(T)), & T \neq 0 \\
0, & T=0
\end{array}\right.
$$
se sigue que $\alpha$ es una parametrización de Puiseaux de $L$. Desde que $\tilde{Z}=\frac{E^{*} Z}{y_{n}^{V}}$
(ver [1]), tenemos que: 


$$
\begin{aligned}
\tilde{\alpha}^{\prime}(T) & =\tilde{Z}(\tilde{\alpha}(T))=\frac{D E^{-1} \circ E(\tilde{\alpha}(T)) Z \circ E(\tilde{\alpha}(T))}{\tilde{\alpha}_{n}(T)^{v}} \\
& =\frac{D E^{-1}(\alpha(T)) Z(\alpha(T))}{\tilde{\alpha}_{n}(T)^{v}}=\frac{X(T)}{\tilde{\alpha}_{n}(T)^{v}}\left(E^{-1} \circ \alpha\right)^{\prime}(T) \\
& =\frac{X(T)}{\tilde{\alpha}_{n}(T)^{v}} \tilde{\alpha}^{\prime}(T)
\end{aligned}
$$

donde $X$ es el campo vectorial holomorfo de la Proposición 1 y $T \in \mathbb{D}_{\in}-\{0\}$.

Concluimos que $X(T)=\tilde{\alpha}_{n}(T)^{v}=T^{m v} \beta(T)^{v}$, con $\beta(0) \neq 0$. Resumimos éstos resultados en el siguiente:

LEMA. Si $\mathcal{F}_{Z} \in \mathcal{D}_{0}^{n}, \tilde{\boldsymbol{p}} \in E^{-1}(0), L$ y $\tilde{L}$ como antes. En las condiciones mencionadas, tenemos que

$$
m_{0}(Z, L)=m_{0}(Z) i_{\tilde{p}}\left(E^{-1}(0), \tilde{L}\right)
$$

Ahora podemos probar que la multiplicidad algebraica de una foliación en $\mathcal{D}_{0}^{n}$, y el índice de intersección son invariantes topológicos. En efecto, sean $\mathcal{F}_{Z}$ y $\mathcal{F}_{Z^{\prime}}$ dos elementos de $\mathcal{D}_{0}^{n}$ tales que $\mathcal{F}_{Z} \sim$ top $\mathcal{F}_{Z^{\prime}}$ por un germen de homeomorfismo $h$. Sea $\tilde{p} \in E^{-1}(0)-S$, donde $S$ es la hipersuperficie de tangencia de $\mathcal{F}_{Z}$, desde que $\tilde{L}$, la hoja de $\tilde{\mathcal{F}}_{Z}$ que pasa por $\tilde{p}$, es transversal al espacio proyectivo $E^{-1}(0)$, tenemos que $i_{\tilde{p}}\left(E^{-1}(0), \tilde{L}\right)=1$. Consideremos $\tilde{U}$ vecindad de $\tilde{p}$ tal que $\left.\tilde{L}\right|_{\tilde{U}} \cap E^{-1}(0)=\{\tilde{p}\}$ y $U$ vecindad de $0 \in \mathbb{C}^{n}$ tal que $E^{-1}[U-\{0\}] \subseteq \tilde{U}$. Si $L=E\left[\left.\tilde{L}\right|_{\tilde{U}}-\{\tilde{p}\}\right]$ entonces $L-\{0\}$ es una hoja de $\mathcal{F}_{Z}$ y $L$ es una subvariedad analítica, irreducible de $U$, invariante por $Z$ y $\operatorname{dim}_{\mathbb{C}} L=1$.

De la definición de $h$, se sigue que $h[L-\{0\}]$ es una hoja de $\mathcal{F}_{Z^{\prime}}$. Sea $U^{\prime}=h[U]$ y $L^{\prime}=\overline{h[L-\{0\}]}$, se sigue del teorema de Remmert-Stein que $L^{\prime}$ es una subvariedad analítica, irreducible de $U^{\prime}$, con singularidad aislada en $0 \in \mathbb{C}^{n}$ y $\operatorname{dim}_{\mathbb{C}} L^{\prime}=1$. Desde que $L^{\prime}-\{0\} \in \mathcal{F}_{Z^{\prime}}$ entonces $E^{-1}[U-\{0\}] \in \mathcal{F}_{Z^{\prime}}$. Por lo tanto $\tilde{L}^{\prime}=E^{-1}[U-\{0\}]$ esta contenido en una hoja de $\mathcal{F}_{Z^{\prime}}$ y $L^{\prime} \cap E^{-1}(0)=\left\{p^{\prime}\right\}$.

Bajo estas condiciones, del lema anterior tenemos que $m_{0}(Z, L)=m_{0}(Z)$ y de la varianza topológica de la multiplicidad a lo largo de $L^{\prime}$ (Proposición 2), se sigue que:

$$
m_{0}(Z)=m_{0}\left(Z^{\prime}, L^{\prime}\right)=m_{0}\left(Z^{\prime}\right) i_{\tilde{p}}\left(E^{-1}(0) ; \tilde{L}\right) .
$$


Por lo tanto $m_{0}(Z)$ es divisible por $m_{0}\left(Z^{\prime}\right)$. Análogamente, si $\tilde{q} \in E^{-1}(0)-S^{\prime}$, donde $S^{\prime}$ es la hipersuperficie de tangencia de $\tilde{\mathcal{F}}_{Z^{\prime}}$, tenemos que $m_{0}\left(Z^{\prime}\right)$ es divisible por $m_{0}(Z)$ y por lo tanto, $m_{0}(Z)=m_{0}\left(Z^{\prime}\right)$.

Más aún, si $\tilde{p} \in E^{-1}(0) \cap S$, de la Proposición 2 y del Lema anterior, tenemos que:

$$
i_{\tilde{p}}\left(E^{-1}(0), \tilde{L}\right)=\frac{m_{0}(Z, L)}{m_{0}(Z)}=\frac{m_{0}\left(Z^{\prime}, L^{\prime}\right)}{m_{0}\left(Z^{\prime}\right)}=i_{\tilde{p}^{\prime}}\left(E^{-1}(0), \tilde{L}^{\prime}\right) .
$$

De esta manera, hemos demostrado el siguiente resultado:

TEOREMA 1. La multiplicidad algebraica de una foliación de $\mathcal{D}_{0}^{n}$ y el índice de intersección, son invariantes topológicos. Más específicamente, sean $\mathcal{F}_{Z}, \mathcal{F}_{Z^{\prime}} \in \mathcal{D}_{0}^{n}, \tilde{p}, \tilde{p}^{\prime}, \tilde{L}$ y $\tilde{L}^{\prime}$ como antes. Si $\mathcal{F}_{Z \sim \text { top }} \mathcal{F}_{Z^{\prime}}$, entonces

(1) $m_{0}(Z)=m_{0}\left(Z^{\prime}\right)$

(2) $\quad i_{\tilde{p}}\left(E^{-1}(0), \tilde{L}\right)=i_{\tilde{p}^{\prime}}\left(E^{-1}(0), \tilde{L}^{\prime}\right)$

Nótese que, de la construcción anterior, podemos definir la función $\tilde{f}$ : $E^{-1}(0) \rightarrow E^{-1}(0)$ por la regla de correspondencia:

$$
\tilde{f}(\tilde{p})=\tilde{p}^{\prime}
$$

Desde que $h$ es un germen de homeomorfismo en $0 \in \mathbb{C}^{n}$, usando $h^{-1}$ es fácil demostrar que $\tilde{f}$ es biyectiva. Más aún, tenemos el sigueinte:

TEOREMA 2. La función $\tilde{f}$ definida por (6) es un homeomorfismo tal que $\tilde{f}[S]=S^{\prime}$, donde $S$ y $S^{\prime}$ son las hipersuperficies de tangencia de las foliaciones $\mathcal{F}_{Z}$ y $\mathcal{F}_{Z^{\prime}}$, respectivamente.

Demostración. Desde que $\tilde{f}$ es una función biyectiva, es suficiente probar que $\tilde{f}$ es continua. Primeramente, probaremos que $\tilde{f}$ es continua sobre $E^{-1}(0)-S$ y luego extenderemos $\tilde{f}$ a una función continua sobre $E^{-1}(0)$.

Sea $\tilde{p} \in E^{-1}(0)-S$, de la definición de $\tilde{f}$ y la parte (2) del Teorema 1, deducimos que $\tilde{f}(\tilde{p})=\tilde{p}^{\prime} \in E^{-1}(0)-S^{\prime}$. sea $\left\{\tilde{p}_{n}\right\}$ una sucesión en $E^{-1}(0)$ tal que $\tilde{p}_{n} \rightarrow \tilde{p}$, probaremos que $\tilde{f}\left(\tilde{p}_{n}\right) \rightarrow \tilde{f}(\tilde{p})=\tilde{p}^{\prime}$. Desde que $\tilde{p}$ y $\tilde{p}^{\prime}$ son puntos regulares de $\mathcal{F}_{Z}$ y $\mathcal{F}_{Z^{\prime}}$, respectivamente, por el Teorema del flujo tubular complejo, existen $\alpha, \alpha^{\prime}>0 ; \tilde{V} \subseteq \tilde{U}, \tilde{V}^{\prime} \subseteq \tilde{U}^{\prime}$ vecindades de $\tilde{p}$ y $\tilde{p}^{\prime}$ (respectivamente) y difeomorfismos holomorfos $\psi$ y $\psi^{\prime}$, donde:

$$
\psi: \mathbb{D}_{\alpha^{\prime}} \times B_{\alpha} \rightarrow \tilde{V}, \psi^{\prime}: \mathbb{D}_{\alpha^{\prime}} \times B_{\alpha^{\prime}} \rightarrow \tilde{V}^{\prime}, B_{\alpha}=\left\{u \in \mathbb{C}^{n-1}:|u|<\alpha\right\}
$$

son tales que, $\psi(0, \overline{0})=\tilde{p}, \psi^{\prime}(0, \overline{0})=\tilde{p}^{\prime}$ y $\psi$ (resp. $\psi^{\prime}$ ) es una conjugaçión analítica local entre $\tilde{Z}$ (resp. $\tilde{Z}^{\prime}$ ) y el campo vectorial constante $\frac{\partial}{\partial t}$, donde $\frac{\partial}{\partial t}(T, u)=(1, \overline{0})$. Más aún, tenemos que $\psi^{-1}\left[E^{-1}(0) \cap \tilde{V}\right]=\{0\} \times B_{\alpha}$ (resp. \left.${\stackrel{\partial}{\psi^{\prime-1}}}^{\prime}\left[E^{-1}(0) \cap \tilde{V}^{\prime}\right]=\{0\} \times B_{\alpha^{\prime}}\right)$. Si $h$ es una conjugación topológica entre $\mathcal{F}_{Z}$ y $\mathcal{F}_{Z^{\prime \prime}}$ 
se sigue que $\tilde{h}=E^{-1} \circ h \circ E$ es una conjugación topológica entre $\mathcal{F}_{Z}$ y $\mathcal{F}_{Z^{\prime}}$ sobre $V-E^{-1}(0)$, donde $\tilde{V}$ es una vecindad de $E^{-1}(0)$. Por lo tanto:

$$
\left.\tilde{h}\right|_{\tilde{V}}: \tilde{V}-E^{-1}(0) \rightarrow \tilde{V}^{\prime}-E^{-1}(0)
$$

es un homeomorfismo y $\tilde{f}=\left.\tilde{h}\right|_{\tilde{\nu} \cap E^{-1}(0)}$.

Definiendo $\tilde{H}=\left(\psi^{\prime}\right)^{-1} \circ h \circ \psi$ y $\tilde{F}=\left(\psi^{\prime}\right)^{-1} \circ \tilde{f} \circ \psi$, se sigue que $\tilde{H}: \mathbb{D}_{\alpha} \times B_{\alpha} \rightarrow \mathbb{D}_{\alpha^{\prime}} \times B_{\alpha^{\prime}}$ y $F:\{0\} \times B_{\alpha} \rightarrow\{0\} \times B_{\alpha^{\prime}}$ tenemos las siguientes propiedades:

- $\tilde{H}$ es continua en $\mathbb{D}_{\alpha}-\{0\} \times B_{\alpha}$.

- $\tilde{H}(T, u)=\left(\tilde{H}_{1}(T, u), \tilde{H}_{2}(u)\right)$.

- $\tilde{H}(0, \overline{0})=(0, \overline{0})$.

- $\tilde{H}_{\tilde{H}}(0, u)=0, \quad \forall u \in B_{\alpha}$.

- $\left.\tilde{H}\right|_{\{0\} \times B_{\alpha}}=\tilde{F}$.

Sin pérdida de generalidad, podemos suponer que la sucesión, $\left\{\tilde{p}_{n}\right\}$ está contenida en $\tilde{V} \cap E^{-1}(0)$, luego $z_{n}=\psi^{-1}\left(\tilde{p}_{n}\right)$, es una sucesión de la forma $z_{n}=\left(0, u_{n}\right)$ con $u_{n} \in B_{\alpha}$ y $u_{n} \rightarrow \overline{0}$. Si $T_{0} \in \mathbb{D}_{\alpha}-\{0\}$ entonces $\left(T_{0}, u_{n}\right) \rightarrow\left(T_{0}, \overline{0}\right)$ y por la continuidad de $\tilde{H}: \tilde{H}\left(T_{0}, u_{n}\right) \rightarrow \tilde{H}\left(T_{0}, \overline{0}\right)$, tenemos $\tilde{H}_{2}\left(u_{n}\right) \rightarrow \tilde{H}_{2}(\overline{0})=\overline{0}$. Se sigue que:

$$
\tilde{F}\left(z_{n}\right)=\tilde{F}\left(0, u_{n}\right)=\tilde{H}\left(0, u_{n}\right)=\left(0, \tilde{H}_{2}\left(u_{n}\right)\right) \rightarrow(0, \overline{0})=\tilde{F}(0, \overline{0}) .
$$

De esta forma, $\tilde{f}$ es contuinua sobre $E^{-1}(0)-S$. Con la finalidad de extender $\tilde{f}$ continuamente a $E^{-1}(0)$, usamos el criterio de Cauchy: Sea $\tilde{p} \in S$, debemos probar que $\forall \varepsilon>0, \exists \delta>0$ tal que $\tilde{p}_{1}, \tilde{p}_{2} \in E^{-1}(0)-S$ con $\left|\tilde{p}_{1}-\tilde{p}\right|<\delta$ y $\left|\tilde{p}_{2}-\tilde{p}\right|<\delta \Rightarrow\left|\tilde{f}\left(\tilde{p}_{1}\right)-\tilde{f}\left(\tilde{p}_{2}\right)\right|<\varepsilon$.

Para $\tilde{p} \in S$ y $\tilde{p}^{\prime}=\tilde{f}(\tilde{p}) \in S^{\prime}$ sea $\alpha, \alpha^{\prime}>0, \tilde{V}, \tilde{V}^{\prime}, \psi$ y $\psi^{\prime}$ como antes, tomando $\alpha$ y $\alpha^{\prime}$ suficientemente pequeños tales que:

$$
\tilde{V}^{\prime} \cap E^{-1}(0) \subseteq\left\{\tilde{q}^{\prime} \in E^{-1}(0):\left|\tilde{q}^{\prime}-\tilde{f}(\tilde{p})\right|<\varepsilon\right\}
$$

y considerando $\delta>0$ tal que $\left\{\tilde{q} \in E^{-1}(0):|\tilde{q}-\tilde{p}|<\delta\right\} \subseteq \tilde{V} \cap E^{-1}(0)$, la hipótesis del criterio de Cauchy se verifica. Así finaliza la demostración del Teorema 2.

Otro resultado que usaremos es que el índice de intersección es constante (salvo en un conjunto de medida de Lebesgue cero) a lo largo de cada componente irreducible de la hipersuperficie de tangencia. Más específicamente, sea $\mathcal{F}_{Z} \in \mathcal{D}_{0}^{n}$ la foliación generada por el campo vectorial $Z=\sum_{i=1}^{n} Z_{i} \frac{\partial}{\partial z_{i}}$ con $Z_{i}=\sum_{k \geq v} Z_{k}^{i}$, en donde los $Z_{k}^{i}$ son polinomios homogéneos de grado $k$ y $m_{0}\left(\mathcal{F}_{Z}\right)=\nu$. Sean 


$$
J_{0}^{v}(Z)=P_{\nu-1} \cdot\left(\sum_{i=1}^{n} z_{i} \frac{\partial}{\partial z_{i}}\right)
$$

el primer Jet no nulo de $Z$ y

$$
S=\left\{\left[z_{1} ; \ldots ; z_{n}\right] \in \mathbb{C} P(n-1): P_{v-1}\left(z_{1}, \ldots, z_{n}\right)=0\right\}
$$

la hipersuperficie de tangencia de $\mathcal{F}_{Z}$. Sabemos que la foliacifon $\tilde{\mathcal{F}}_{Z}$ es generada por el campo $\tilde{Z}$ el cual, en la carta $\tilde{U}_{n}$ de $\tilde{\mathbb{C}}^{n}$ (estamos usando las notaciones de [1]), se expresa como:

$$
\tilde{Z}=\sum_{i=1}^{n} \tilde{z}_{i} \frac{\partial}{\partial y_{i}}
$$

donde:

$$
\left\{\begin{array}{l}
\tilde{Z}_{i}\left(y_{1}, \ldots, y_{n}\right)=\sum_{k \geq v} y_{n}^{k-v}\left[Z_{k+1}^{i}(\hat{y})-y_{i} Z_{k+1}^{n}(\hat{y})\right] \\
\tilde{Z}_{i}\left(y_{1}, \ldots, y_{n}\right)=P_{v-1}(\hat{y})+\sum_{k \geq v+1} y_{n}^{k-\nu} Z_{k}^{n}(\hat{y})
\end{array}\right.
$$

e $\hat{y}=\left(y_{1}, \ldots, y_{n-1}, 1\right)$.

Dado $\tilde{p}=\left(y_{1}^{0}, \ldots, y_{n-1}^{0}, 0\right) \in E^{-1}(0) \cap \tilde{U}_{n}$, consideremos $\tilde{L}$ la hoja de $\tilde{\mathcal{F}}_{Z}$ que pasa por $\tilde{p}$, la cual puede ser parametrizada por la curva analítica:

$$
\tilde{\alpha}=\left(\tilde{\alpha}_{1}, \ldots, \tilde{\alpha}_{n}\right):\left(\mathbb{D}_{\varepsilon}, 0\right) \rightarrow(\tilde{L}, \tilde{p})
$$

donde

$$
\left\{\begin{array}{l}
\tilde{\alpha}^{\prime}(T)=\tilde{Z}(\tilde{\alpha}(T)), \text { i.e. } \tilde{\alpha}_{i}^{\prime}(T)=\tilde{Z}_{i}(\tilde{\alpha}(T)), \\
\tilde{\alpha}(0)=\tilde{p} .
\end{array}\right.
$$

Por lo tanto $\tilde{\alpha}_{n}^{\prime}(0)=\tilde{A}_{n}\left(y_{1}^{0}, \ldots, y_{n-1}^{0}, 0\right)=P_{v-1}\left(y_{1}^{0}, \ldots, y_{n-1}^{0}, 1\right)$. Concluimos que:

$$
i_{\tilde{p}}\left(E^{-1}(0), \tilde{L}\right)>1 \Leftrightarrow \tilde{p} \in S
$$

Dado $\tilde{p} \in S$, calcularemos $\tilde{\alpha}^{\prime \prime}(0)$. De (9) tenemos que: $\tilde{\alpha}_{n}^{\prime \prime}(T)=\sum_{i=1}^{n} \frac{\partial \tilde{Z}_{n}}{\partial y_{i}}(\tilde{\alpha}(T)) \tilde{\alpha}_{i}^{\prime}(T)$, luego 


$$
\begin{aligned}
\tilde{\alpha}_{n}^{\prime \prime}(0) & =\sum_{i=1}^{n-1} \frac{\partial \tilde{Z}_{n}}{\partial y_{i}}(\tilde{p}) \tilde{Z}_{i}(\tilde{p}) \\
& =\sum_{i=1}^{n-1} \frac{\partial \tilde{P}_{v-1}}{\partial z_{i}}\left(\tilde{y}_{0}\right)\left[Z_{v+1}^{i}\left(\tilde{y}_{0}\right)-y_{i}^{0} Z_{v+1}^{n}\left(\tilde{y}_{0}\right)\right] \\
& =\sum_{i=1}^{n-1} \frac{\partial \tilde{P}_{v-1}}{\partial z_{i}}\left(\tilde{y}_{0}\right) Z_{v+1}^{i}\left(\tilde{y}_{0}\right)-Z_{v+1}^{n}\left(\tilde{y}_{0}\right) \sum_{i=1}^{n-1} y_{i}^{0} \frac{\partial \tilde{P}_{v-1}}{\partial z_{i}}\left(\tilde{y}_{0}\right)
\end{aligned}
$$

donde $\tilde{y}_{0}=\left(y_{1}^{0}, \ldots, y_{n-1}^{0}, 1\right)$. Por lo tanto:

$$
\tilde{\alpha}_{n}^{\prime \prime}(0)=\sum_{i=1}^{n-1} \frac{\partial \tilde{P}_{v-1}}{\partial z_{i}}\left(y_{1}^{0}, \ldots, y_{n-1}^{0}, 1\right) Z_{v+1}^{i}\left(y_{1}^{0}, \ldots, y_{n-1}^{0}, 1\right)
$$

Definimos el polinomio homogéneo $Q_{2 v-1}$ de građo $2 v-1$ como:

$$
Q_{2 v-1}=d P_{v-1}\left[Z_{v+1}\right]=\sum_{i=1}^{n} \frac{\partial P_{v-1}}{\partial z_{i}} Z_{v+1}^{i}
$$

y denotamos por $S^{*}$ a la hipersuperficie algebraica generada por $Q_{2 v-1}$, i.e.

$$
S^{*}=\left\{\left[z_{1} ; \ldots ; z_{n}\right] \in \mathbb{C} P(n-1): Q_{2 v-1}\left(z_{1}, \ldots, z_{n}\right)=0\right\}
$$

de (10) deducimos que:

$$
i_{\tilde{p}}\left(E^{-1}(0), \tilde{L}\right)>2 \Leftrightarrow \tilde{p} \in S \cap S^{*} .
$$

Note que los puntos singulares de la hipersuperficie de tangencia $S$ están contenidos en $S^{*}$. Sin embargo, $S \cap S^{*}$ contiene otros puntos $\tilde{p}$ los cuales no son singularidades de $S$ pero $i_{\tilde{p}}\left(E^{-1}(0), \tilde{L}\right)>2$. En efecto, si consideramos por ejemplo el campo vectorial holomorfo

$$
Z\left(z_{1}, z_{2}, z_{3}\right)=\left(z_{1} z_{3}, z_{2} z_{3}+z_{1}^{3}, z_{3}^{2}+z_{1}^{2} z_{2}-z_{2}^{3}\right),
$$

se tiene que $\mathcal{F}_{Z} \in \mathcal{D}_{0}^{3}, m_{0}(Z)=2$ y el polinomio homogéneo de grado 1 asociado a $Z$ es $P_{1}\left(z_{1}, z_{2}, z_{3}\right)=z_{3}$. Entonces, la hipersuperficie de tangencia $S$ de $\mathcal{F}_{Z}$ viene dada por:

$$
S=\left\{\left[z_{1} ; z_{2} ; z_{3}\right] \in \mathbb{C} P(2): z_{3}=0\right\}
$$

además $Q_{3}\left(z_{1}, z_{2}, z_{3}\right)=d P_{1}\left[Z_{3}\right]\left(z_{1}, z_{2}, z_{3}\right)=z_{1}^{2} z_{2}-z_{2}^{3}$, luego:

$$
S^{*}=\left\{\left[z_{1} ; z_{2} ; z_{3}\right] \in \mathbb{C} P(2): z_{1}^{2} z_{2}-z_{2}^{3}=0\right\}
$$




$$
S \cap S^{*}=\{[1 ; 1 ; 0],[1 ;-1 ; 0],[1 ; 0 ; 0]\}
$$

Como se prueba en [4], en los tres puntos de $S \cap S^{*}$ el índice de intersección es 3 y si $\tilde{p} \in S-S^{*}$, entonces $i_{\tilde{p}}\left(E^{-1}(0), \tilde{L}\right)=2$.

El ejemplo anterior nos muestra que el índice de intersección es constante en casi todo punto de cada componente irreducible de la hipersuperficie de tangencia $S$. Más específicamente, sea $\mathcal{F}_{Z} \in \mathcal{D}_{0}^{n}$ con $m_{0}\left(\mathcal{F}_{Z}\right)=\nu$ tal que $J_{0}^{v}(Z)=P_{\nu-1}\left(\sum_{i=1}^{n} z_{i} \frac{\partial}{\partial z_{i}}\right)$.

Desde que $P_{v-1}$ es un polinomio homogéneo de $n$ variables, puede ser factorizado:

$$
P_{v-1}=F_{1}^{r_{1}} \cdots F_{l}^{r_{l}}
$$

donde los $F_{j}(1 \leq j \leq l)$, son polinomios homogéneos irreducibles de grado $\operatorname{deg}\left(F_{j}\right)=g_{j}$. Esta factorización es única salvo factores constantes no nulos. De acuerdo a la factorización (13), podemos escribir la hipersuperficie de tangencia

$$
S=\left\{\left[z_{1} ; \ldots ; z_{n}\right] \in \mathbb{C} P(n-1): P_{v-1}\left(z_{1}, \ldots, z_{n}\right)=0\right\}
$$

como:

$$
S=r_{1} S_{1}+\ldots+r_{l} S_{l}
$$

donde:

$$
S_{j}=\left\{\left[z_{1} ; \ldots ; z_{n}\right] \in \mathbb{C} P(n-1): F_{j}\left(z_{1}, \ldots, z_{n}\right)=0\right\}
$$

Las hipersuperficies algebraicas $S_{j}(1 \leq j \leq l)$ son llamadas las componentes irreducibles de $S$, mientras que los números enteros positivos $g_{j}$ y $r_{j}$ son llamados respectivamente, el grado y la multiplicidad de $S_{j}$ en $S$. Es claro que, el grado y la multiplicidad de $S_{j}$ en $S$ satisfacen la ecuación:

$$
\sum_{j=1}^{l} r_{j} g_{j}=v-1
$$

De manera análoga a (11), definimos los polinomios homogéneos:

$$
G_{j}=d F_{j}\left[Z_{v+1}\right]=\sum_{i=1}^{n} \frac{\partial F_{j}}{\partial z_{i}} Z_{v+1}^{i}
$$


y denotamos por $S_{j}^{*}$ a la hipersuperficie algebraica:

$$
S_{j}^{*}=\left\{\left[z_{1} ; \ldots ; z_{n}\right] \in \mathbb{C} P(n-1): G_{j}\left(z_{1}, \ldots, z_{n}\right)=0\right\} .
$$

Note que los puntos singulares de la hipersuperficie $S_{j}$ están contenidos en $S_{j}^{*}$. Bajo éstas condiciones, tenemos el siguiente resultado, cuya demostración puede ser encontrarla por el lector en [4]:

TEOREMA 3. Con las notaciones introducidas anteriormente, sea $\tilde{p} \in S_{j}-S_{j}^{*}$ tal que $\tilde{p} \notin S_{i}, 1 \leq i \leq l, i \neq j$. Entonces $i_{\tilde{p}}\left(E^{-1}(0), \tilde{L}\right)=r_{j}+1$.

\section{EL RESULTADO PRINCIPAL}

Todos los resultados dados en la sección anterior son válidos en cualquier dimensión $n$. Sin embargo, cuando $n=3$, podemos detectar la existencia de otros invariantes topológicos los cuales, como veremos, están fuertemente relacionados con el primer Jet no nulo de campo $Z$ que génera a la foliación.

Sea $\mathcal{F}_{Z} \in \mathcal{D}_{0}^{3}$ generada por $Z=\sum_{i=1}^{3} Z_{i} \frac{\partial}{\partial z_{i}}$ con $Z_{i}=\sum_{k \geq v} Z_{k}^{i},(1 \leq i \leq 3)$ donde los $Z_{k}^{i}$ son polinomios homogéneos de grado $k, Z_{v}^{i}=z_{i} P_{v-1}$ y $m_{0}(Z)=v$. En este caso, la hipersuperficie de tangencia $S$ es una curva algebraica en $\mathbb{C} P(2)$ definida anteriormente por $S=\left\{\left[z_{1} ; z_{2} ; z_{3}\right] \in \mathbb{C} P(2): P_{v-1}\left(z_{1}, z_{2}, z_{3}\right)=0\right\}$. Usando las notaciones de la sección anterior, si $P_{v-1}$ es un polinomio no irreducible, entonces puede ser factorizado por

$$
P_{v-1}=F_{1}^{i_{i}} \cdots F_{l}^{r^{\prime}}
$$

y

$$
S=r_{1} S_{1}+\cdots+r_{l} S_{l}
$$

donde $S_{j}=\left\{\left[z_{1} ; z_{2} ; z_{3}\right] \in \mathbb{C} P(2): F_{j}\left(z_{1}, z_{2}, z_{3}\right)=0\right\},(1 \leq j \leq l)$ son las componentes irreducibles de $S$. Empleamos las siguientes notaciones:

$\#(S)=l:$ número de componentes irreducibles de $S$.

$r(S)=\left\{r_{1}, \ldots, r_{l}\right\}$, donde $r_{j}$ es la multiplicidad de $S_{j}, \forall 1 \leq j \leq l$.

$g(S)=\left\{g_{1}, \ldots, g_{l}\right\}$, donde $g_{j}$ es el grado de $S_{j}, \forall 1 \leq j \leq l$.

Para cada componente irreducible $S_{j}$ de $S$, asociaremos la curva algebraica:

$$
S_{j}^{*}=\left\{\left[z_{1} ; z_{2} ; z_{3}\right] \in \mathbb{C} P(2): G_{j}\left(z_{1}, z_{2}, z_{3}\right)=0\right\},(1 \leq j \leq l)
$$

donde $G_{j}$ es el polinomio homogéneo de grado $g_{i}+v$ definido por

$$
G_{j}=\sum_{i=1}^{3} \frac{\partial F_{j}}{\partial z_{i}} Z_{v+1}^{i}
$$

Es claro que los puntos singulares de la curva $S_{j}$ están contenidos en $S_{j}^{*}$. 
Del Teorema 3 se sigue que, si $\tilde{p} \in S_{j} \cap S_{j}^{*}$, entonces $i_{\tilde{p}}\left(E^{-1}(0), \tilde{L}\right)>r_{j}+1$, donde $\tilde{L}$ es la hoja de $\tilde{\mathcal{F}}_{Z}$ que pasa por $\tilde{p}$. Una propiedad importante de las curvas $S_{j}$ y $S_{j}^{*}$ es que ellas no tienen componentes comunes. Más aún, tenemos la siguiente:

PROPOSICION 3. En las condiciones anteriores, tenemos que para todo $1 \leq j \leq l, S_{j} \cap S_{j}^{*}$ es un conjunto finito que tiene $g_{j}\left(g_{j}+v\right)$ elementos.

DEMOSTRACION. Procediendo por contradicción, suponga que $S_{j}$ y $S_{j}^{*}$ tienen una componente en común. Desde que $S_{j}$ es irreducible, tenemos que:

$$
G_{j}=\sum_{i=1}^{3} \frac{\partial F_{j}}{\partial z_{i}} Z_{v+1}^{i}=F_{j} Q
$$

donde $Q$ es un polinomio homogéneo de grado $v$. Consideremos el siguiente sistema de ecuaciones homogéneas:

$$
\mid \begin{aligned}
& P_{v-1}(z)=0 \\
& z_{2} Z_{v+1}^{1}(z)-z_{1} Z_{v+1}^{2}(z)=0 \\
& z_{3} Z_{v+1}^{1}(z)-z_{1} Z_{v+1}^{3}(z)=0 \\
& z_{3} Z_{v+1}^{2}(z)-z_{2} Z_{v+1}^{3}(z)=0
\end{aligned}
$$

Sea $z_{0}=\left(z_{1}^{0}, z_{2}^{0}, z_{3}^{0}\right)$ un punto de $\mathbb{C}^{3}$ tal que $F_{j}\left(z_{0}\right)=0$ y $z_{1}^{0} Z_{v+1}^{1}\left(z_{0}\right)-z_{1}^{0} Z_{v+1}^{2}\left(z_{0}\right)=0$. Podemos asumir, sin pérdida de generalidad, que $z_{0}$ es un punto regular de $F_{j}$, más aún, $\frac{\partial F_{j}}{\partial z_{3}}\left(z_{0}\right) \neq 0$. De (19) y la fórmula de Euler, no es difícil probar que:

$$
\begin{aligned}
& \frac{\partial F_{j}}{\partial z_{3}}\left(z_{0}\right)\left(z_{3}^{0} Z_{v+1}^{1}\left(z_{0}\right)-z_{1}^{0} Z_{v+1}^{3}\left(z_{0}\right)\right)= \\
= & -\frac{\partial F_{j}}{\partial z_{2}}\left(z_{0}\right)\left(z_{2}^{0} Z_{v+1}^{1}\left(z_{0}\right)-z_{1}^{0} Z_{v+1}^{2}\left(z_{0}\right)\right)=0
\end{aligned}
$$

y

$$
\begin{aligned}
& \frac{\partial F_{j}}{\partial z_{3}}\left(z_{0}\right)\left(z_{3}^{0} Z_{v+1}^{2}\left(z_{0}\right)-z_{2}^{0} Z_{v+1}^{3}\left(z_{0}\right)\right)= \\
= & -\frac{\partial F_{j}}{\partial z_{1}}\left(z_{0}\right)\left(z_{2}^{0} Z_{v+1}^{1}\left(z_{0}\right)-z_{1}^{0} Z_{v+1}^{2}\left(z_{0}\right)\right)=0
\end{aligned}
$$

De este modo $z_{0} \neq 0 \in \mathbb{C}^{3}$ es una solución de (20), pero ésto implica (ver [1]) que $\mathcal{F}_{Z} \notin \mathcal{D}_{0}^{3}$ lo cual es una contradicción. De esta manera, la Proposición 3 sigue del Teorema de Bezout. 
Consideremos $\mathcal{F}_{Z}$ y $\mathcal{F}_{Z}, \in \mathcal{D}_{0}^{3}$ tales que, $\mathcal{F}_{Z \sim \text { top }} \mathcal{F}_{Z^{\prime}}$. Por la parte (1) del Teorema 1 , tenemos que $m_{0}(Z)=m_{0}\left(Z^{\prime}\right)=v$. Sea $S$ (resp. $S^{\prime}$ ) la curva de tangencia de $\mathcal{F}_{Z}$ (resp. $\mathcal{F}_{Z^{\prime}}$ definida por el polinomio homogéneo $P_{v-1}$ (resp. $\left.P_{v-1}^{\prime}\right)$ el cual es factorizado como $P_{v-1}=F_{1}^{\prime i} \cdots F_{l}^{r_{i}}$ (resp. $\left.P_{v-1}^{\prime}=\left(F_{1}^{\prime}\right)^{r_{1}^{\prime}} \cdots\left(F_{l}^{\prime}\right)^{r_{i}^{\prime}}\right)$ donde $\operatorname{deg}\left(F_{j}\right)=g_{j}, 1 \leq j \leq l$ (resp. $\left.\operatorname{deg}\left(F_{j}^{\prime}\right)=g_{j}^{\prime}, 1 \leq j \leq l^{\prime}\right)$. Entonces $S=r_{1} S_{1}+\cdots+r_{l} S_{l}$ (resp. $\left.S^{\prime}=r_{1}^{\prime} S_{1}^{\prime}+\cdots+r_{l}^{\prime}, S_{l}^{\prime}\right)$. Estamos en condiciones de enunciar y demostrar nuestro resultado principal:

TEOREMA. Consideremos $\mathcal{F}_{Z}$ y $\mathcal{F}_{Z^{\prime}} \in \mathcal{D}_{0}^{3}$. Con las notaciones anteriores, si $\mathcal{F}_{Z \sim \text { top }} \mathcal{F}_{Z}$, entonces

(1) $\#(S)=\#\left(S^{\prime}\right)$.

(2) $r(S)=r\left(S^{\prime}\right)$.

(3) $g(S)=g\left(S^{\prime}\right)$.

En donde $\#(S)$ es el número de elementos del conjunto $S$.

Demostración. Desde que $\mathcal{F}_{Z \sim \text { top }} \mathcal{F}_{Z^{\prime}}$ del Teorema 4, existe un homeomorfismo $\tilde{f}: \quad E^{-1}(0) \rightarrow E^{-1}(0)\left(E^{-1}(0)=\mathbb{C} P(2)\right)$ tal que $\tilde{f}[S]=S^{\prime}$. Primero, consideremos el caso cuando $S$ es irreducible. Afirmamos que $S^{\prime}$ también es irreducible. En efecto, suponiendo por contradicción que $S^{\prime}=S_{1}^{\prime} \cup S_{2}^{\prime}$, del Teorema de Bezout se sigue que $S_{1}^{\prime} \cap S_{2}^{\prime}$ consiste de un número finito de puntos $\left\{\tilde{p}_{1}^{\prime}, \ldots, \tilde{p}_{N}^{\prime}\right\}\left(N=\operatorname{deg}\left(S_{1}^{\prime}\right) \cdot \operatorname{deg}\left(S_{2}^{\prime}\right)\right)$. Denotemos $\tilde{p}_{j}=\tilde{f}^{-1}\left(\tilde{p}_{j}^{\prime}\right), 1 \leq j \leq N \mathrm{y}$ consideremos $\hat{S}=S-\left\{\tilde{p}_{1}, \ldots, \tilde{p}_{N}\right\}$ y $\hat{S}^{\prime}=S^{\prime}-\left\{\tilde{p}_{1}^{\prime}, \ldots, \tilde{p}_{N}^{\prime}\right\}$ con la topología inducida de $\mathbb{C} P(2)$. Bajo estas condiciones, tenemos que $\left.\tilde{f}\right|_{\hat{S}}: \hat{S} \rightarrow \hat{S}^{\prime}$ es un homeomorfismo entre espacios topológicos, donde $\hat{S}$ es conexo pero $\hat{S}^{\prime}$ no lo es, lo cual es una contradicción. Para el caso general, aplicamos el argumento anterior a cada componente irreducible $S_{j}$ de $S$. Concluimos que $S$ y $S^{\prime}$ tienen igual número de componentes irreducibles, es decir $\#(S)=\#\left(S^{\prime}\right)$, lo cual prueba la parte (1).

Denotemos $f\left[S_{j}\right]=S^{\prime}{ }_{j}, \forall 1 \leq j \leq l$. Desde que $S_{j} \cap S_{j}^{*}$ es finito $\forall \mathcal{F}_{Z^{\prime}} \in \mathcal{D}_{0}^{3}$, (ver Proposición 3), podemos elegir un punto $\tilde{p} \in S_{j}-\left(S_{j}^{*} \cup\left(\bigcup_{i=1, i \neq j} S_{i}\right)\right)$ tal que, $\tilde{p}^{\prime}=\tilde{f}(\tilde{p}) \in S_{j}^{\prime}-\left(\left(S_{j}^{*}\right)^{*} \cup\left(\bigcup_{i=1, i \neq j}^{\prime} S_{i}^{\prime}\right)\right)$. Sea $\tilde{L}$ (resp. $\tilde{L}^{\prime}$ ) la hoja de $\tilde{\mathcal{F}}_{Z}$ (resp. $\tilde{\mathcal{F}}_{Z^{\prime}}$ ) que pasa por $\tilde{p}$ (resp. $\left.\tilde{p}^{\prime}\right)$. De la parte (2) del Teorema 1 y el Teorema 3, tenemos que:

$$
r_{j}+1=i_{\tilde{p}}\left(E^{-1}(0), \tilde{L}\right)=i_{\tilde{p}^{\prime}}\left(E^{-1}(0), \tilde{L}^{\prime}\right)=r_{j}^{\prime}+1
$$

entonces $r_{j}=r_{j}^{\prime} ; \forall 1 \leq j \leq l$. Por lo tanto $r(S)=r\left(S^{\prime}\right)$, lo cual prueba la parte 2).

Finalmente, desde que tenemos un homeomorfismo de $\mathbb{C} P(2)$ en $\mathbb{C} P(2)$ tal que $S$ es homeomórficamente mapeado en $S^{\prime}$, técnicas usadas en [5], podemos probar que $g_{j}=g_{j}{ }_{j}, \forall 1 \leq j \leq l$. Esto finaliza la prueba del Teorema C. 
Finalmente, debemos mencionar que el teorema anterior generaliza los resultados obtenido por M. Klughertz (ver Teorema I.2 de [11]), en dimensión compleja 2, al caso 3-dimensional.

\section{BIBLIOGRAFIA}

[1] R. Benazic, Caracterización de singualridades dicríticas en foliaciones de dimensión uno. PESQUIMAT, Vol I, No 1, (1998), p. 73-81.

[2] R. Benazic, invarianza Topológica del índice de intersección. PESQUIMAT, Vol IV, No1, (2001), p. 31-38.

[3] R. Benazic, Sobre el Número de Milnor. PESQUIMAT, Vol III, No1, (2000), p. 115.

[4] R. Benazic, El indice de intersección a lo largo de un conjunto de tangencia. PESQUIMAT, Vol IV, N², (2001), p. 55-65.

[5] E. Brieskorn, H. Knörrer, Plane algebraic curves. Birkhäuser - Verlag (1986).

[6] C. Camacho, L. Neto, P. Sad, Topological Invariants and Equidesingularization for Holomorphic Vector Fields. J. Differential Geometry 20, (1984), p. 143-174.

[7] D. Cerveau, J. Matter, Formes Intégrables Holomorphes Singulières. Asterisque, 97, (1982).

[8] E. Chirka, Complex Analytic Sets. MIA, Kluwer Academic Publishers. Dordrecht, Boston, London (1989).

[9] P. Griffiths, J. Harris, Principles of Algebraic Geometry. WileyIntersciencie, New York, (1978).

[10] R. Gunning, H. Rossi, Analytic functions of several complex variables. Prentice-Hall, Englewood Cliffs, N.J., (1965).

[11] M. Klughertz, Feuilletages holomorphes a singularité isolee ayant une infinite de courbes intégrables. These, Université Paul.Sabatier - Toulouse, (1988).

[12] J. Mattei, R. Moussu, Holonomie et intégrales premières. Ann. Sci. Ecole. Norm. Sup. (4) 13 (1980) p. 469-523.

[13] H. Whitney, Complex Analytic Varieties. Addison-Wesley Publishing Company, (1972). 